\title{
Solidarité et singularité au sein des équipes sportives
}

\author{
Pascal Duret \\ Muriel Augustini
}

Qu'est-ce qu'une équipe si ce n'est le mythe exalté d'une entente idéale ? Les célébrations incessantes de "I'esprit d'équipe » représentent des axiomes enchantés qui imposent l'image d'une immédiate et indéfectible solidarité. II suffirait de franchir les portes d'un vestiaire pour que se dissolvent aussitôt les prétentions individuelles, les appétits égoïstes et que le "nous " supplante les " je ". En rupture avec ces clichés, le terrain montre en pratique, au sein des équipes, des tensions entre entraide et concurrence. Pour gagner sa place, supporter les hauts et les bas du championnat, s'ajuster aux exigences de l'entraîneur, la coopération entre équipiers s'accompagne de rivalités. Comme la compétition ne s'arrête pas aux portes de l'équipe, la cohésion ne va pas de soi. Aujourd'hui, devenir un "bon coéquipier " revient à réussir à rester solidaire tout en tirant son épingle du jeu. L'abnégation de l'individu au service du collectif n'est plus acceptée sur un mode sacrificiel mais à condition de faire du collectif une ressource pour les individus.

La rationalisation de la performance nécessite une division croissante du travail d'encadrement des joueurs, à la base de la création de nouveaux métiers. Au tandem entraîneur/joueurs sont venus se rattacher le coach (responsable de la préparation mentale des joueurs), le préparateur

Pascal Duret, professeur, directeur du DIMPS EA 4075. pascal.duret@univ-reunion.fr Muriel Augustini, maître de conférences, DIMPS EA 4075. muriel.augustini@univreunion.fr 
physique (responsable de la forme physique des joueurs et en particulier de leur récupération en état de fatigue), le diététicien (en charge de l'optimisation des apports nutritionnels), le responsable de la communication (chargé des relations entre les joueurs et les médias), I'agent recruteur (qui aide l'entraîneur à bâtir un effectif en repérant des joueurs d'un bon rapport " qualité/prix " sur le marché des mutations). La performance est donc le produit d'un système qui englobe de plus en plus d'acteurs au fur et à mesure que I'on s'élève dans la hiérarchie sportive. Dans le club de quartier, I'entraîneur fera figure d'homme orchestre mobilisant à lui seul des compétences qui seront confiées à un ensemble de spécialistes dans les grands clubs professionnels. Clubs de quartier et clubs de haut niveau vivent donc des réalités parallèles et cloisonnées. Qu'ont-elles de comparable ? Sans doute les questions touchant aux conditions de l'efficacité de la relation entre joueurs et entraîneur. L'obéissance aveugle à l'entraîneur est-elle indispensable ? Si oui, est-elle suffisante pour obtenir les meilleures performances ? L'amitié est-elle une forme d'autorité qui contraint les individus plus sûrement que la discipline ? Comment évoluent les relations entre joueurs et entraîneurs dans le temps et en fonction des disciplines sportives?

Les réponses à ces questions ne trouvent guère d'unité théorique. En 2006, la revue Psychology of Sport and Exercise publie un numéro spécial dédié à l'état de la question des relations interpersonnelles en sport. Jowett et Wylleman y mettent en avant le manque de connaissances relatives aux équipes sportives. Dans cette même revue, Poczwardowski, Barott et Jowett (2006) remarquent que les rares études existantes privilégient les analyses individuelles. Afin de dépasser cette limite, ils proposent de développer des analyses comparatives intersport. Toujours en 2006, un numéro thématique de la revue International Journal of Sport and Exercise Psychology est consacré à l'intérêt pour l'étude des équipes sportives des théories issues du courant de la "Team cognition" (Ward, Eccles, 2006). La critique majeure adressée à cette approche en psychologie du sport est, là encore, la focalisation sur l'expertise individuelle des sportifs alors même que le domaine étudié est celui des sports collectifs. L'alternative proposée par les auteurs est de placer la coordination des activités individuelles au premier plan des analyses. Nous allons essayer de prendre en compte ces attentes en nous focalisant sur l'analyse de l'activité des joueurs en action afin de saisir les transformations de leurs manières de se coordonner.

DE GRANDES ÉVOLUTIONS DANS LES RELATIONS ENTRE ENTRAÎNEUR ET JOUEURS

\section{Du commandement à la négociation}

La première de ces évolutions concerne les liens entre entraîneur et joueurs, et entre coéquipiers. La norme d'autorité s'est profondément 
transformée. Fini le temps où l'entraîneur tout-puissant pouvait exiger une obéissance inconditionnelle à ses directives. L'entraîneur doit souvent, aujourd'hui, justifier voire négocier ses choix. L'attitude de Raymond Domenech durant la phase finale de la Coupe du monde de football 2010 peut, dans ce cadre, constituer un cas d'école tant les joueurs ont, à de multiples reprises, refusé l'exécution de ses consignes ${ }^{1}$. Ainsi, I'ex-entraîneur des Bleus s'est illustré par son grand écart entre, d'une part, la surdité aux remarques des joueurs (quand il tentait d'imposer ses choix par absolutisme) et, d'autre part, le renoncement à toute autorité quand il adoptait les solutions proposées par les "joueurs cadres ${ }^{2}$ ».

Qui dit négociation suppose que l'entraîneur ne soit pas le seul dépositaire des principes de justice qui régulent l'équipe. Au sein du collectif se déploie donc tout un jeu de concurrences, où chacun cherche des alliés parfaitement disposés à s'entre-épauler pour peser sur les choix de l'entraîneur et gérer l'accès au terrain en constituant des sortes de petits groupes de pression. Être collectif, ce n'est pas bâillonner son individualité, mais simplement réussir à contribuer à un sens de la justice partagé (c'est-à-dire réussir à se mettre d'accord sur les formes légitimes de domination des uns sur les autres). Ainsi, dans toute équipe s'entrechoquent des principes de justice contradictoires. On peut, par exemple, repérer classiquement dans les équipes nationales l'opposition entre le principe de respect de l'ancienneté et le principe de respect des jeunes talents. Les plus avancés dans la carrière chercheront à convertir leur nombre de sélections en prestige. À l'opposé, les plus jeunes pourront faire valoir leur appartenance à tel ou tel grand club (au football, jouer au Real ou à Arsenal donne plus de crédit qu'évoluer dans un club de milieu de tableau du championnat français). Le choc des principes de justice, plus qu'une façon ponctuelle de régler un litige, constitue une manière indispensable d'élaborer une culture commune. II est donc nécessaire que cette négociation soit entretenue de manière continue (et pas seulement en temps de crise) pour parvenir à des compromis permettant de stabiliser l'esprit du groupe.

Pour faciliter la négociation, l'entraîneur aménage le cadre (spatial et temporel) des discussions : autour du tableau noir durant les séances dédiées à la tactique (et non dans les vestiaires durant l'immédiat après-

1. Ainsi, avant même la crise entre Anelka et Domenech à la mi-temps du match France-Mexique, Thierry Henry avait fait remarquer à l'entraîneur que les joueurs ne voyaient pas où il voulait en venir.

2. Les joueurs "cadres " étaient ceux qui ont sauvé Domenech, menacé de limogeage après la contre-performance des Bleus à l'Euro de 2008. Le miraculé devenait leur débiteur et son managérat ressemblait à un arrangement au nom de l'intérêt mutuel bien compris. Quel que fût leur niveau de performance, les joueurs qui soutinrent Domenech à I'issue de I'Euro 2008 restèrent quasi inamovibles. Non seulement ils avaient une place sur le terrain, mais ils semblaient en mesure de choisir leur poste. 
match saturé d'émotions), durant le débriefing les lendemains de compétitions à l'occasion des visionnages de matchs enregistrés (Mouchet, 2005 ; Duret, 2011). Négocier ne signifie pas que tout soit négociable. Ainsi, pour l'entraîneur, constituer la liste des joueurs titulaires et remplaçants est une prérogative qui lui revient en propre. De même, pour I'entraîneur, la présence à l'entraînement des joueurs n'est pas négociable (par contre, l'allégement des charges de travail en raison de la fatigue accumulée ou de séquelles de blessure l'est). De leur côté, pour la plupart des joueurs, I'accès au terrain (ou la relégation sur le banc des remplaçants) en fonction du mérite est un principe très peu négociable ${ }^{3}$. Le fait qu'un joueur hors de forme reste sur le terrain alors qu'un remplaçant en pleine possession de ses moyens ne rentre pas à sa place constitue une injustice interprétée par les joueurs soit en termes de " caprice " de I'entraîneur (pointant son "immaturité "), soit en termes de "despotisme " (pointant son arbitraire). Un des premiers accords passés entre entraîneurs et joueurs concerne donc les limites du négociable ${ }^{4}$.

\section{Des normes pour le temps court du match et pour le temps long de la vie du groupe}

La négociation porte aussi sur le domaine des normes de la vie du groupe. Toute équipe n'est pas, en effet, qu'un ensemble de joueurs mobilisés le temps du match, c'est aussi une communauté amenée à partager - même sur un temps minimal - un mode de vie. Traditionnellement au rugby, faire la fête était, par exemple, de mise pour célébrer une victoire. La troisième mi-temps est encore souvent donnée par les joueurs comme indispensable à la cohésion. Ces "bringues " vécues comme des moments de réduction des tensions constituent des expériences communes favorables à l'admiration mutuelle où le groupe s'héroïse aussi à travers ses excès festifs. Pourtant, les entraîneurs les présentent souvent aujourd'hui comme des dépenses physiques néfastes à l'efficacité compétitive. Ils dénoncent du coup l'irresponsabilité des joueurs qui, s'y livrant, mettent en péril le résultat sportif. Aussi s'abstenir de toute fête a été donné par Marc Liévremont comme impérieuse règle de vie au XV de France lors de la dernière Coupe du monde de rugby en

3. Certes, on I'a vu, I'ancienneté dans le club ou encore la notoriété vont être des contre-arguments face au principe du mérite, mais finalement très mineurs. Ainsi il a été mis un terme à la présence de Sébastien Chabal sur le terrain lors des matchs avec l'équipe nationale, son niveau de jeu ayant été jugé insuffisant bien que son image reste très médiatisée et " marketisée ".

4. Dans certains sports, le principe de propriété du corps est acquis. L'entraîneur de volley-ball ou de handball ne peut guère proposer à ses joueurs de prendre tel ou tel produit dopant. Dans d'autres sports, la santé entre dans le domaine du négociable et un entraîneur de cyclisme ou d'haltérophilie pourra directement faire pression sur ses poulains pour les inciter à prendre des produits dopants. 
Nouvelle-Zélande. Après leur victoire contre les joueurs du pays de Galles en demi-finale de la compétition, les joueurs se sont trouvés confrontés à un entraîneur satisfait mais hostile à toute forme de troisième mi-temps. Les joueurs ont bravé cet interdit pour montrer à Marc Liévremont que s'il voulait conserver de l'autorité, il devait prendre en compte leurs attentes. Ainsi, le troisième ligne biarrot Imanol Harinordoquy confia à Thibault Perrin : «À partir de ce moment, je n'ai plus attaché d'importance à ce que disait Marc Lièvremont. On a décidé de se prendre en main. Cette aventure était la nôtre. Ça devait être la belle histoire de trente mecs. II fallait qu'on $\mathrm{s}^{\prime}$ affranchisse de lui ${ }^{5}$. "

Qui dit négociation suppose enfin, par définition, que chacun des négociateurs dispose $\mathrm{d}^{\prime}$ assez de pouvoir pour obliger l'autre à tenir compte de ses propres positions. Tant que l'entraîneur bénéficie d'une situation de pouvoir absolu, pourquoi engagerait-il un processus de négociation ? Or, cette position de domination sans partage est aujourd'hui largement remise en cause, en particulier dès que les résultats ne sont plus au rendez-vous. L'épée de Damoclès, représentée par le risque de limogeage, menace alors immédiatement l'entraîneur. En sport professionnel en particulier, sponsors et actionnaires attendent un retour quasi immédiat sur investissement des sommes qu'ils ont injectées dans le club. Ainsi, les investisseurs qataris acheteurs en 2011 du PSG manifestent des objectifs incompatibles avec des résultats en demi-teintes. II aura suffi au PSG de subir trois défaites en début de saison pour que ses dirigeants envisagent, dès le mois novembre ( $c$ 'est-à-dire avant même le milieu de saison), le remplacement de l'entraîneur Antoine Kambouaré. Les joueurs peuvent, en se contentant de prestations " au petit trot ", accélérer l'exécution de la sentence qui condamne leur $\operatorname{coach}^{6}$. Au contraire, s'ils n'économisent pas leurs efforts et font l'impossible pour l'emporter, ils montrent aux dirigeants leur attachement à l'entraîneur. Les joueurs disposent donc de moyens de pression.

\section{Effets de la professionnalisation}

Sous I'effet de la professionnalisation, I'ancien couple « joueur/ entraîneur " fait place aujourd'hui à un nouveau tandem " joueur/agentimpresario "(Desbordes et coll., 2004). Plus le processus de professionnalisation d'une discipline est avancé, plus les joueurs deviennent autonomes vis-à-vis de leur entraîneur. Dans les sports individuels les plus professionnalisés (I'équipe se concevant alors comme l'ensemble des membres concourant à la production de la performance), I'entraîneur

5. "Lièvremont ne lui manquera pas ", Le Figaro, 30 octobre 2011, p. 11.

6. "L'avenir de Kambouaré est de plus en plus sombre ", France Inter, journal des sports, 28 novembre 2011. Les noms d'Ancelotti, de Makélé puis de Benitez sont avancés pour lui succéder sur les sites de sport comme Goal1.fr, comsofoot. com, ou encore lesdessousdusport.fr 
n'est souvent qu'un prestataire de service. En tennis ou en Formule 1, il est convoqué et remercié au gré des besoins définis par le sportif. II n'a plus rien du mentor tout-puissant sur la carrière des joueurs ; il est I'humble employé au service de son riche employeur. Ce constat ne vaut certes pas de la même manière pour tous les sports ${ }^{7}$.

L'exigence de résultats ne pèse pas uniquement sur l'entraîneur mais aussi sur les joueurs eux-mêmes. Les rivalités sont d'autant plus exacerbées que les équipes comptent aujourd'hui deux, voire trois joueurs prétendant au même poste. La compétition interne au club est perceptible même dans les catégories de jeu intermédiaires. La coupure entre un sport amateur débonnaire et un sport professionnel à la compétition dure apparaît, sur ce point, de moins en moins tranchée. Ainsi, dès le niveau " régional ", le renforcement des effectifs et l'élévation du niveau de jeu des remplaçants font que les places ne sont jamais acquises de manière définitive. Moins les statuts établis rendent étanches le cloisonnement entre "joueurs cadres " et "joueurs de second rôle " et plus l'équipe est concurrentielle. Les "stars " sont les exceptions qui confirment la règle de la mise en concurrence (Duret, Leroyer, 2002). Mais, il peut arriver que même Lionel Messi, dont le talent prodigieux est unanimement reconnu, se retrouve provisoirement sur le banc des remplaçants du Barça $^{8}$ quand il est hors de forme. Disposer d'une équipe à l'effectif des plus fournis permet également à l'entraîneur d'économiser ses joueurs en mettant ses vedettes au repos sur les matchs jugés faciles, réduisant ainsi le risque de blessures occasionnées par la fatigue.

En outre, les journalistes tiennent, eux aussi, un rôle important dans la mise en concurrence des joueurs en leur attribuant des notes individuelles après chaque match. Ces évaluations, bien qu'elles s'appuient sur des données chiffrées (nombre de buts, nombre d'interceptions, nombre de passes décisives, nombre de ballons perdus, nombre de tirs cadrés ou non...), sont toujours dénoncées par ceux qui en pâtissent comme un simple signe de copinage entre tel journaliste et tels joueurs les mieux notés. Cela a pour effet d'accroître les tensions au sein des équipes.

\section{Une expertise partageable}

La norme d'autorité s'est également modifiée à cause du quasiévanouissement de la frontière entre "ceux qui savent " et ceux qui sont là "pour les écouter ". Les équipiers, mêmes spécialisés, sont de plus en plus capables d'analyser l'action de leurs coéquipiers investis dans d'autres rôles. Ce constat est particulièrement flagrant en voile où

7. Dans les sports les moins médiatisés ou encore dans les sports de combat reposant sur une tradition de transmission autoritaire (comme au judo ou au karaté), les interactions entraîneurs/entraînés opèrent un renforcement des rituels et de la discipline (Riff, Avanzini, 1997).

8. Par exemple le 10 septembre 2011 contre la Real Sociétad. 
skippers et équipiers étaient liés dans un rapport hiérarchique faisant des premiers les commandants des seconds réduits à des rôles de simples exécutants. Les skippers détenaient seuls l'autorité pour prendre les grandes décisions (fixer le cap, réduire ou au contraire envoyer de la toile, virer de bord). Cette autorité se manifestait à travers des séquences d'ordres régissant précisément chaque manœuvre. À la question "Paré à virer ? ", les coéquipiers ne pouvaient répondre que "paré ", mais en aucun cas par exemple : "Je préférerais qu'on attende qu'il y ait un peu moins de vent. " Le pouvoir absolu du barreur s'est réduit, toutefois inégalement, dans les courses en dériveurs (470,505, Fliyng Dutchman) et dans les courses de haute mer en quillards. Dans les courses hauturières (comme les transatlantiques), des générations successives de skippers, celle de Francis Chichester et Eric Tabarly, puis celle de Mike Birch et Olivier de Kersauzon, enfin celle de Michel Desjoyeaux ou Vincent Riou, ont réussi à conserver presque intacte la tradition du capitaine " seul maître à bord après Dieu ${ }^{9}$ ". Ces grands skippers ont tous fait leurs preuves en solitaire. C'est de cette exposition au danger extrême qu'ils puisent leur légitimité de commandement quand ils naviguent en équipe. Inversement, dans les régates de dériveurs, il est désormais admis qu'un équipage mené par un barreur expert ne devient pas forcément une équipe experte. La performance suppose, dans ce type d'embarcations, des ajustements en cours d'action en vue de collaborer à la prise de décision. Saury et Durand expliquent que, même lorsqu'ils se trouvent face à des alternatives simples en apparence (comme continuer sur le même bord ou virer), les régatiers doivent appuyer leurs choix tactiques sur des prises d'informations complexes ${ }^{10}$ (Saury, Durand, 1998). Mais leur mode de prise de décision n'est en rien celui des joueurs d'échecs confortablement installés dans leur fauteuil, pesant à l'envi le pour et le contre. En régate, les équipiers doivent, tout en s'activant pour faire avancer leur bateau, saisir les opportunités et décider dans I'instant. Les régatiers poursuivent toujours et en même temps deux buts : optimiser la vitesse du bateau et prendre les meilleures décisions tactiques. Pour utiliser les " deux cerveaux à bord » (Saury, 2011), il est nécessaire de renoncer à la division traditionnelle des rôles assurant au seul barreur le rôle de tacticien. Aujourd'hui, le mode de répartition des tâches décisionnelles dans les équipages suppose une complémentarité entre barreur et équipier. Dans les sections de louvoyage ${ }^{11}$, par exemple, le barreur doit s'occuper de la vitesse du bateau et contrôler l'espace

9. Dans des courses comme le Vendée globe, la Route du rhum ou la Transat anglaise.

10. Ils doivent prendre en considération les variations de direction du vent, la présence et l'évolution des courants, la position relative des équipages adverses, les intentions des équipages adverses.

11. C'est-à-dire quand le bateau doit remonter au vent pour aller tourner derrière une bouée. 
proche ; I'équipier, lui, est plus disponible pour saisir l'environnement lointain. Mais quand le bateau passe sous le vent, les rôles s'inversent. Cette alternance a plusieurs conséquences relationnelles : la communication est permanente, les statuts sont plus équilibrés. La complémentarité des deux équipiers et leur polyvalence s'accompagnent aussi d'une compétence accrue à la critique de l'activité de l'autre équipier. Échanger sur des différences d'appréciation est une source d'optimisation de la performance, mais aussi une source potentielle d'accroissement des risques de conflits. En course, la confiance que se portent les équipiers est mise à l'épreuve par des événements imprévus dès qu'ils entraînent des interprétations divergentes de la situation. Sur le huis clos des bateaux ${ }^{12}$, chacun des coéquipiers peut remettre en cause le consensus sur un mode explicite ou implicite (par exemple donner un petit coup de barre imperceptible quand il avait été décidé de ne pas changer de cap, ou étarquer discrètement la voile quand la décision était de ne pas y toucher). Ces tentatives de dissimulation sont souvent décelées par I'autre équipier ${ }^{13}$, ce qui avive les tensions. Du coup, la collaboration nécessite moins un accord parfait que I'élaboration d'une culture commune dans la gestion des désaccords.

Le constat de compétences élargies permettant au joueur d'apprécier finement I'activité de ses coéquipiers est d'actualité également en rugby où, traditionnellement, avants et arrières vivaient dans deux sousgroupes peu ouverts l'un à l'autre ; chacun développant ses propres critères d'excellence et ses propres formes de reconnaissance. Christian Pociello avait détaillé les oppositions entre "rugby de tranchée ", et "rugby champagne "(Pociello, 1983). Des avants, hommes de devoir, était attendu qu'ils repoussent les adversaires en mêlée ou dans les regroupements, sans chercher à jouer eux-mêmes les ballons âprement gagnés. Ce "rugby de tranchée " reposait sur les qualités viriles d'une phalange massive guidée par les demis (surnommés les "cornacs ", "les poissons pilotes ") chargés d'exploiter le travail d'extraction de ballons mené par les lignes avants. Les arrières, de leur coté, pouvaient, dans un "rugby champagne ", jouer aux toréadors en déroutant les avants par leurs crochets et leurs folles cavalcades. Au jeu groupé « en force " des avants s'opposait le jeu déployé en finesse des arrières. Lors des palabres de comptoir d'après-match, les commentaires enflammés

12. L'activité de l'équipage reste hors de portée de l'entraîneur durant le temps de la course.

13. Celui-ci peut considérer la transgression bénigne, insupportable ou intolérable. Rappelons la différence entre l'insupportable et l'intolérable. L'insupportable ne le devient qu'après répétition. L'insupportable est l'aboutissement d'un processus inscrit dans le temps. L'insupportable fait l'objet d'une gradation : d'abord, c'est agaçant, puis fatigant, puis épuisant, puis exaspérant et finalement " insupportable ". Trop, c'est trop. Au contraire, " l'intolérable " naît d'une faute ponctuelle, une et une seule suffit, mais une faute dans le domaine du non-négociable. 
soulignaient l'ampleur des différences de styles de jeu : les arrières revenant volontiers sur les "valises " infligées à leurs vis-à-vis, les avants du "fauteuil d'orchestre " (première ligne) se félicitant de n'avoir pas cédé un pouce de terrain en mêlée. Aujourd'hui, cette dichotomie radicale est dépassée. La rationalisation du jeu (en particulier défensif et son très haut degré d'exigence physique) a conduit à l'émergence de morphotypes extrêmement athlétiques, et cela quel que soit le poste occupé. L'avant empâté comme l'arrière filiforme sont quasi introuvables dans le rugby professionnel actuel (Charlot, 2011). Les quinze joueurs doivent être capables de tenir leur rôle dans le rideau défensif. Ces transformations morphologiques ont permis d'estomper la division du travail sportif entre avants et arrières. William Servat, première ligne, peut très bien porter le ballon à la main tel un centre et Maxime Médard, arrière, n'hésite pas à aller percuter, si nécessaire, les avants adverses. Les joueurs gagnent en polyvalence et ont une compréhension plus fine du jeu des autres lignes. Les coachs longtemps recrutés parmi les lignes arrières (Pierre Villepreux, Jacques Fourroux) ont laissé place au coach issu des avants (avant d'entraîner le XV de France, Marc Lièvremont a joué troisième ligne à I'USAP). Pour lui, la fonction de l'entraîneur est non seulement de faire progresser les joueurs dans leur rôle, mais aussi $d^{\prime}$ 'aiguiser leurs capacités d'appréciation de l'activité mise en jeu par leurs partenaires.

\section{UNE CARRIÈRE PENSÉE PAR SOI-MÊME}

La seconde grande évolution concerne l'autonomie grandissante des joueurs qui ne lient plus leurs destins que dans un temps limité à l'avance (celui de leur contrat). La mobilité, en particulier dans le sport professionnel, devient une condition et un atout clé de la réussite. La mutation change de fonction. Elle ne sert plus à marquer un désaccord. Elle n'est plus une offense faite par un joueur à " son " équipe et à " sa " ville, mais une épreuve décisive et incontournable pour qui souhaite avancer dans sa carrière. Une saison réussie peut propulser un joueur à un nouveau niveau d'ambition. Le succès dans un club sert alors de marchepied pour accéder à un club plus prestigieux. Zinedine Zidane avait fait ses premières armes à Cannes puis, repéré par Bordeaux, il évolua quelques saisons avec les Girondins avant d'être recruté par la Juventus de Turin, pour finalement revêtir le maillot des Galactiques du Real de Madrid (apothéose de sa carrière). Didier Deschamps, actuel entraîneur de l'équipe de France, est passé en tant que joueur du FC Nantes à l'Olympique de Marseille, puis à la Juventus de Turin et enfin au club anglais de Chelsea. Les joueurs jaugent leur progression à leur valeur marchande lors des transferts. La durée moyenne passée par un joueur du championnat français dans un même club diminue régulièrement : de six ans en 1980, elle s'est réduite à quatre en 1994, puis à deux ans et trois mois en 2000 (Duret, Trabal, 
2000) ${ }^{14}$. Changer de club est perçu comme une nécessité même au rugby, sport où pourtant les valeurs de fidélité et d'attachement au club sont restées très vivaces jusqu'à la professionnalisation de la discipline durant les années 1995-2000 ${ }^{15}$. II n'y avait, au rugby, de héros que fidèles à la couleur du maillot. Hier, le joueur en partance était qualifié de " mercenaire ", stigmatisation blessante à l'extrême dans un monde où le profit monétaire arrivait loin derrière les liens d'amitié et de fidélité au club. II passait pour un " traître " aux siens. Aujourd'hui, le souci de porter les couleurs et les valeurs de la communauté dont on est issu n'est plus d'actualité. Dans le rugby professionnel, I'appartenance à l'équipe suppose même l'absence de culpabilité à la quitter. Le changement devient une valeur en soi ${ }^{16}$. L'ancienneté dans un club, hier source de respect, est aujourd'hui un signe d'impuissance (à évoluer ailleurs). L'antériorité ne fait plus loi, le respect des anciens se perd. L'individualité ne peut se développer sur la base de la seule appartenance. Cette évolution avive la tension entre autonomie et interdépendance. Le joueur a encore besoin de savoir qu'il est bien intégré au groupe, mais pour s'en différencier. L'appartenance est devenue une simple toile de fond au point que certains joueurs peuvent "stratégiquement " préférer choisir un club faible où ils seront très visibles et médiatisés que de prendre le risque de muter dans un très grand club où leur place, vu le niveau de concurrence interne, ne serait pas garantie.

\section{Projets communs à durée limitée et recomposition des équipes}

L'appartenance n'est pas exclusive, elle ne s'oppose pas à des liens sociaux ouverts. Le joueur n'existe pas uniquement à travers l'attachement à son club du moment mais par l'ensemble de son réseau relationnel où figurent des joueurs d'autres clubs. L'autonomie grandissante est donc synonyme d'affranchissement. La tension appartenance/ affranchissement est particulièrement nette chez les cyclistes quand, lors d'une échappée, ils sont amenés à faire équipe avec des coureurs qui ne portent pas le même maillot qu'eux. Comment se recomposent

14. Les entraîneurs cherchent à imposer un rallongement des contrats signés avec des joueurs de plus en plus jeunes (pour spéculer sur les valeurs montantes puisées à même les centres de formation), alors que les joueurs, tant qu'ils s'estiment en progression, cherchent à en réduire la durée (ce n'est qu'en fin de carrière qu'un sportif essaiera d'obtenir un contrat le plus long possible).

15. Ces valeurs ont dans les faits disparu, mais une condition du succès de cette discipline, c'est qu'elle continue à les mettre en scène pour le public en entretenant I'image d'une famille.

16. L'importance et le sens du flux migratoire des joueurs disent aussi la santé de la discipline. Les All Blacks de Nouvelle-Zélande ou les Wallabies d'Australie accueillis dans les clubs français ne sont plus des exceptions. De même, les meilleurs joueurs français s'exportent à leur tour dans les championnats de I'hémisphère Sud. 
alors ces équipes d'un jour? De quelles marges de manœuvre disposent (ou s'octroient) les coureurs face aux plans de course de leur directeur d'équipe ? Traditionnellement, lorsqu'ils font partie d'une échappée d'un jour, les équipiers doivent encore faire le jeu de leur leader. Selon la position du chef d'équipe et de ses rivaux au classement général, les fuyards devront mener l'échappée avec plus ou moins d'intensité. Si le leader de l'équipe est en tête ou bien placé au classement général, la fonction assignée aux équipiers dans l'échappée est d'obliger les leaders des autres équipes restés dans le peloton à " porter le poids de la course " (c'est-à-dire à se fatiguer pour essayer de rattraper l'échappée). Or cette stratégie est remise en cause aujourd'hui. Les fuyards peuvent passer, dans I'instant, des alliances dites " de circonstance " visant à saisir euxmêmes leur chance. Tout en restant en concurrence, ces équipiers coalisés prennent des libertés par rapport aux consignes de leurs directeurs sportifs pour se mettre en situation d'emporter une victoire personnelle. Les coureurs refusent $d$ 'ailleurs le port d'oreillettes pour ne pas être reliés en permanence à leur entraîneur et se soustraire ainsi plus aisément à ses consignes de course. Dans ce cas, les coureurs échappés "roulent ensemble "; ils reforment, le temps de l'échappée, une nouvelle équipe éphémère. Cette collaboration s'opère le plus souvent dans la méfiance, chacun pouvant suspecter les autres de ne pas jouer le jeu et de continuer à " rouler pour leur équipe ". L'indice majeur pour vérifier la loyauté d'un coureur au groupe des échappées est la régularité de ses prises de relais. L'échappée, pour réussir, suppose en effet une adaptation collective à l'effort à fournir face à la résistance aérodynamique (le coureur de tête dépense une énergie bien supérieure à celle de ceux qui lui " sucent la roue " et profitent de son sillage). À tour de rôle, les coureurs passent en tête pour fendre le vent. Refuser de prendre le relais ("sauter son tour ") est immédiatement interprété comme une volonté de ruiner l'échappée ${ }^{17}$. Les organisateurs de courses, qui édictent les règles de l'épreuve, sont en droit de réprimer les arrangements entre coureurs d'équipes différentes. Dans les faits, ils ne les sanctionnent jamais, et du coup, en les tolérant, ils les encouragent. En effet, les échappées imprévisibles et incontrôlables contribuent au spectacle. Ces "trêves " et " pactes de non-agression " font partie du paysage cycliste (Chiotti, 2001, p. 67).

\section{ÊTRE SINGULIER TOUT EN ÉTANT COLLECTIF}

Enfin, la troisième évolution qui touche l'équipe sportive concerne l'exigence de singularité des individus qui la composent. Traditionnellement le " bon équipier " devait savoir s'effacer, mettre ses forces au service exclusif du collectif. Raymond Poulidor avait, par exemple, la réputation

17. Sauf dans les tout derniers kilomètres de la course où cela peut vouloir aussi signifier " je me repose pour gagner le sprint final ". 
d'être un peu trop égoïste. Pourtant, dans le Tour de France 1967, il va réussir à modifier durablement son image. La victoire semble enfin sourire à cet " éternel second ", quand, dans une étape de montagne légendaire (Divonne-les-Bains/Briançon), il va sacrifier ses chances de I'emporter en allant au secours de Roger Pingeon, son chef d'équipe en difficulté. Goddet, dans L'Équipe, se fait le chantre de ce beau geste : "Poulidor exemplaire s'est mis au service de son camarade décroché du peloton. II n'y eut dans son esprit aucune incertitude... Poulidor repêchait Pingeon comme un noyé, lui réoffrant l'appui de sa roue arrière comme d'une bouée. Et le visage de Poupou, éclairé de bonté, de sollicitude, se dirigeait vers son camarade "(Goddet, L'Équipe, 11 juillet 1967, p. 3). À partir de ce jour et jusqu'à la fin de sa carrière, L'Équipe surnomma Poulidor "l'ange gardien ", construisant ainsi, à l'opposé de son image initiale, la figure d'un champion hybride entre héros et saint. La dualité œuvre/personne a longtemps reposé sur deux économies contradictoires. Si le comble de l'admiration pour l'œuvre tient dans des performances données pour uniques, le summum de la célébration de l'homme porte au contraire sur sa grandeur face à la défaite et au sacrifice.

En cyclisme, la composition du groupe idéal a longtemps été présentée par les directeurs d'équipe comme nécessitant un leader au fort caractère, épaulé par des coureurs complémentaires - grimpeurs, rouleurs, sprinteurs -, tous prêts à s'épanouir dans le dévouement. Analysant les récits autobiographiques de champions cyclistes célèbres, Buisine montre les comportements attendus du leader : il doit toujours, quelles que soient les circonstances, se comporter "en véritable patron " (Buisine, 2010). Eddy Merckx, Bernard Hinault ${ }^{18}$, Lance Armstrong sont des figures emblématiques de ce type de leaders assumant pleinement $d^{\prime}$ imposer des ordres à des équipiers à leur service exclusif. Pour Cyrille Guimard, coureur puis directeur de course respecté, une relation saine entre le " patron " et ses équipiers repose sur des rôles différenciés mais impliquant un investissement total des uns et des autres. Les équipiers s'exténuent pour favoriser la victoire du leader, mais celui-ci doit rendre I'aide reçue en n'étant pas lui-même avare de ses efforts. Aujourd'hui, la multiplication des courses inscrites au calendrier sportif fait qu'un leader ne peut pas toutes les briguer, ce qui suppose une réorganisation des rôles. La tactique du "tous pour un " où tous les équipiers n'avaient comme seul objectif que la réussite du leader est loin d'aller de soi. À sa place, les directeurs des grosses équipes recrutent plusieurs leaders spécialisés, alors que ceux des petites formations laissent les équipiers libres de tenter leur chance dans les courses qui ne sont pas au planning du leader. Buisine montre que le discours des équipiers met à distance

18. Pourtant, Bernard Hinault fut un leader à part en acceptant l'introduction dans son équipe La vie claire d'un second " chef de file ", Greg Lemond. Mais ce partage du pouvoir annonçait plus une transition qu'une cohabitation. 
celui des directeurs d'équipes vantant systématiquement l'esprit de sacrifice. Ils ne se satisfont guère de passer toute leur carrière " à protéger le leader ", " à aller au charbon ", sans la moindre gloire. Le rôle d'équipier, quand il se limite à celui de "porteur d'eau ${ }^{19}$ ", induit un renoncement peu enviable. Pourtant, les coéquipiers se font eux-mêmes les garants de I'impératif de loyauté. Ils ne font donc pas que s'émanciper, pour partie ils perpétuent l'emprise qui pèse sur eux. Chacun, à la fois, résiste et impose l'emprise de la norme de dévouement au service du leader. Les équipiers souhaitent à la fois se soustraire à l'emprise des directeurs de courses, mais ne se privent pas, en même temps, de juger sévèrement ceux qui " exagèrent " en ne pensant qu'à eux. Penser à soi ne veut pas dire ne penser qu'à soi. II ne s'agit donc pas d'une remise en cause radicale et indifférenciée de l'équipe en temps qu'acteur collectif. Si, de manière globale, le rôle d'équipier est déprécié, c'est qu'il ne permet pas l'expression de soi. Ceux qui l'exercent envisagent deux grandes stratégies : la première revient à considérer leur emploi actuel comme une simple étape en vue de décrocher un contrat plus valorisé de "lieutenant ", voire celui de leader (quitte à ce que ce soit dans une équipe de moindre envergure). La seconde consiste à redéfinir leur tâche pour qu'elle puisse laisser s'exprimer leur singularité. Quelles sont les aspirations à la singularité que les coureurs jugent légitimes ? L'exemple type de la négation de soi insupportable aujourd'hui à l'équipier est son recrutement sur des critères essentiellement morphologiques. En effet, dans chaque équipe, un des équipiers devra être la doublure du leader (avoir les mêmes caractéristiques morphologiques) pour pouvoir en cas de défaillance mécanique lui offrir son vélo sans perdre de temps en réglage.

\section{Le collectif comme ressource de l'individualité}

Gagner une étape quand ils sont en position pour le faire (au lieu de s'effacer systématiquement pour laisser la place à leur leader) est une aspiration majeure des coureurs équipiers. II s'agit pour eux d'accéder au quart d'heure de célébrité qu'évoquait Andy Warhol, mais aussi d'affirmer leur capacité. L'abnégation au service du collectif ou du chef d'équipe ne suffit plus aujourd'hui à satisfaire coureurs et joueurs, ils attendent en retour du collectif qu'il soit une ressource pour construire leur singularité. Certes au sein d'une équipe tous n'ont pas le même statut. Seuls ceux qui accèdent au statut de "star " hors du commun peuvent se mettre en avant même en commettant des fautes sans déconstruire leur image mais, au contraire, en la confortant dans son exception (le coup de boule de Zidane). Toutefois tous souhaitent devenir de grands singuliers.

19. Cette fonction de "porteur d'eau " consiste à faire la navette entre la tête du peloton et la voiture de son directeur sportif pour rechercher ce dont les leaders ont besoin. 
Comment rester un "bon coéquipier " tout en affirmant son individualité ? Comment rester solidaire tout en tirant son épingle du jeu ? Ces questions interrogent le cadre moral de l'équipe et sa mutation dans une société singulariste (Martucelli, 2010). Les équipes sportives reflètent, en ce sens, l'avènement de l'exigence de singularité à l'intérieur de la société individualisante (Martucelli, Singly, 2009). L'individualisme suppose que les liens sociaux entre joueurs ne soient pas seulement déterminés par leurs obligations statutaires. L'individu est alors défini par ses choix. II est celui qui n'est pas d'abord déterminé par son appartenance. L'exigence de singularité va plus loin que celle de l'individualisme. Elle suppose, en effet, que le culte de l'individu passe par une performance permettant de se rendre visible au sein même de son équipe. Le culte du grand singulier repose moins sur l'intégration que sur la différenciation.

\section{CONCLUSION}

Les formes relationnelles au sein des équipes sont étroitement liées au niveau de jeu et au processus de professionnalisation des disciplines sportives. Pourtant au terme de cet article on peut dégager quelques invariants du "faire équipe ". Ainsi "faire équipe ", c'est mobiliser un groupe pour parvenir à un but commun explicite dont l'atteinte suppose l'interdépendance d'activités individuelles ajustées entre elles. Cette mobilisation passe pour certaines actions par la concertation (par exemple les choix tactiques). D'autres actions supposent simplement I'application immédiate d'une norme (par exemple, riposter aux coups par les coups en rugby). "Faire équipe ", c'est donc, quel que soit le niveau et quelle que soit la spécialité, viser un but commun explicite. Une équipe s'établit sur la base d'un projet commun. À la différence d'effets produits par la sommation d'actions menées sans visée collective consciente, les membres de l'équipe sportive doivent s'engager vers un but connu et recherché par tous. À l'inverse des équipes dans le monde du travail ou des équipes scientifiques qui peuvent collaborer à distance en ne se rencontrant que très épisodiquement, la coactivité sur un même lieu est une donnée fondamentale en sport. "Faire équipe " suppose donc la mise en présence et l'interdépendance. Cette interdépendance n'est pas uniquement celle des comportements imposés par la tactique mais aussi celle des émotions (le doute est contagieux, mais à l'inverse l'exaltation l'est aussi). Dire que les membres de l'équipe sont interdépendants revient à dire qu'ils sont tenus de coopérer. Dire qu'ils sont autonomes revient à dire en même temps qu'ils peuvent choisir de ne pas le faire. Contrairement à la vision naïve et enchantée faisant de la coopération le seul mode relationnel au sein de l'équipe sportive, on peut affirmer sans risque que l'activité collective est un entrelacs de relations coopératives et concurrentielles. L'emboîtement des joueurs pensés comme les pièces d'un puzzle collectif ne s'opère évidemment pas par miracle, 
mais suppose un travail d'ajustement. Entraîner n'est pas qu'une activité sur autrui mais encore et surtout une activité de travail avec autrui. En définitive, l'équipe sportive est un groupe d'appartenance défini par ses objectifs et sa coactivité. Celle-ci n'est pas restreinte au moment du match, mais s'étend à tous les moments partagés entraînant la production de règles de vie.

Dans l'ensemble des sports, on peut globalement retrouver les mêmes attentes de renforcement des individualités à l'intérieur même des équipes. La cohésion n'est plus un postulat de départ qui gommerait les individualités, mais elle implique une activité d'ajustement pour les articuler. La norme d'autorité était la gomme qui effaçait I'individu. Mais les relations entre entraîneurs et entraînés se sont profondément transformées. Les consignes tactiques et les prises de décision, loin d'être dictées de manière inflexible, font souvent I'objet de négociations, d'évaluations mutuelles et de régulations collectives en cours d'action. II est donc essentiel de s'intéresser à cette incessante activité de coordination pour envisager ses effets sur les différentes tensions qui traversent les groupes sportifs. La tension majeure concerne aujourd'hui I'opposition entre solidarité et individualisme. Les conduites d'effacement des individualités et de "sacrifice " au profit du collectif entrent en conflit avec toutes celles où les joueurs savent tirer leur épingle du jeu.

\section{BIBLIOGRAPHIE}

BROMBERGER, C. 1995. Le match de football, Paris, MSH.

BUISINE, S. 2010. Faire le métier de cycliste, Sarrebruck, Éditions universitaires européennes.

CHARLOT, V. 2011. "Le rugby professionnel : socialisation et évolution des "normes" ", dans R. Bercot, S. Divay, C. Gadéa (sous la direction de), Les groupes professionnels en tension, Toulouse, Octarès, p. 88-101.

CHIOTTI, J. 2001. De mon plein gré !, Paris, Calmann-Lévy.

DARBON, S. (sous la direction de). 1999. Rugby d'ici, une manière d'être au monde, Paris, Autrement.

Desbordes, M. ; OHL, F. ; TRIBOU, G. 2004. Marketing du sport, Paris, Economica.

DURET, P. 2008. Sociologie du sport, Paris, PUF, coll. "Que sais-je ? "

DURET, P. 2009. Sociologie de la compétition, Paris, Armand Colin.

DURET, P. (sous la direction de). 2011. Faire équipe, Paris, Armand Colin.

DURET, P. ; LEROYER, P. 2002. "Les hauts et les bas des stars sportives », Le débat, $\mathrm{n}^{\circ} 121$, p. 112-125.

DURET, P. ; TRABAL, P. 2001. Le sport et ses affaires, Paris, Métailié.

ELIAS, N. 1991. La société des individus, Paris, Fayard.

JOWETT, S. ; WYLLEMAN, P. 2006. "Interpersonal relationships in sport and exercise settings : crossing the chasm ", Psychology of Sport and Exercise, $\mathrm{n}^{\circ} 7$, p. 119-123.

MARTUCELLI, D. 2010. La société singulariste, Paris, Armand Colin. 
MARTUCelli, D. ; Singly, F. (de). 2009. Les sociologies de l'individu, Paris, Armand Colin.

MOUCHET, A. 2005. "Subjectivity in the articulation between strategy and tactics in team sports : an example in rugby ", Italian Journal of Sport Sciences, $\mathrm{n}^{\circ} 12,1, \mathrm{p} .24-33$.

POCIELLO, C. 1983. Le rugby ou la guerre des styles, Paris, Métailié.

POCZWARDOSKI, A. ; BAROTT, J. E. ; JOWETT, S. 2006. "Diversifying approaches to research on athlete-coach relationships ", Psychology of Sport and Exercise, $\mathrm{n}^{\circ}$ 7, p. 125-142.

RIFF, J. ; AVANZINI, G. 1997. "Autonomie et dépendance des judokas à l'égard du coaching pendant les combats : une question de confiance ?", Communication aux $5^{e}$ JORESCAM, Toulouse.

SAOUTER, A. 2000. Être rugby, Paris, MSH.

SAURY, J. 2011. "Comment les équipages experts en voile prennent-ils leurs décisions tactiques au cours des régates ? ", dans P. Duret (sous la direction de), Faire équipe, Paris, Armand Colin.

SAURY, J. ; DURAND, M. 1998. "Practical knowledge in expert coaches : on-site study of coaching in sailing ", Research Quarterly for Exercise and Sport, $\mathrm{n}^{\circ}$ 69, p. 254-266.

SAURY, J. ; SÈVE, C. (coord. par). 2004. L'entraînement, Paris, Éditions Revue EPS.

VIGARELlO, G. 2002. Du jeu ancien au show sportif, Paris, Le Seuil.

VIgARELLO, G. (sous la direction de). 2004. L'esprit sportif aujourd'hui, Paris, Universalis.

WARD, P. ; ECCLES, D. W. 2006. " A commentary on team cognition and expert teams : emerging insights into learning and performance for exceptional teams ", International Journal of Sport and Exercise Psychology, $\mathrm{n}^{\circ} 4$, p. 463-483.

PASCAL DURET, MURIEL AugustinI, SOlIDARITÉ ET SINGULARITÉ AU SEIN DES ÉOUIPES SPORTIVES

\section{RÉSUMÉ}

Rompant avec les clichés idéalisant une solidarité donnée comme évidente au sein des équipes sportives, l'article montre que la cohésion est le fruit d'un travail d'ajustement entre les membres qui la composent. Les décisions, loin d'être dictées de manière inflexible, font souvent l'objet de négociations, d'évaluations mutuelles et de régulations collectives en cours d'action. D'où trois évolutions majeures : la première concerne la transformation de l'autorité de l'entraîneur. II puise aujourd'hui son autorité de sa capacité d'écoute. La seconde renvoie à la plus grande autonomie des joueurs. Les joueurs sont moins définis par leur fidélité dans le temps à un même club que par un projet commun qui les rend interdépendants pour une durée limitée. Enfin la troisième évolution concerne l'exigence de singularité des coéquipiers. Ceux-ci ne veulent rester solidaires, et servir le collectif, que si en retour ils peuvent affirmer leur individualité.

MOTS-CLÉS

Équipe sportive, solidarité, ajustement, autorité, autonomie. 
PASCAL DURET, MURIEL AUgUSTINI, SOlIDARITY AND SINGULARITY WITHIN SPORT TEAMS

\section{ABSTRACT}

Breaking with the pictures of an idealized solidarity given as evidence in sport teams, the article shows that cohesion is the result of a work adjustment of their members. Decisions, rather than being dictated in an inflexible way, are often subjects of negotiations, mutual evaluations and collective regulations during the action. Three major developments concern the way to be in a team ; the first concerns the transformation of the authority of the coach. Today he draws his authority from his ability to listen. The second refers to the greater autonomy of the players. Players are less defined by their allegiance to the same club than by a project that makes them interdependent for a limited time. The third change concerns the request of singularity. Players do not want to serve the collective if in return they can assert their individuality.

KEYWORDS

Sport teams, solidarity, adjustment, authority, autonomy. 